\title{
Mass Loss from a Stretching Semitransparent Sheet of Liquid Tin
}

\author{
Bo Liu $\odot,{ }^{1,2}$ Dmitry Kurilovich $\odot,{ }^{3}$ Hanneke Gelderblom, ${ }^{4}$ and Oscar O. Versolato ${ }^{1, *}$ \\ ${ }^{1}$ Advanced Research Center for Nanolithography (ARCNL), Science Park 106, Amsterdam 1098 XG, Netherlands \\ ${ }^{2}$ Department of Physics and Astronomy, and LaserLaB, Vrije Universiteit Amsterdam, De Boelelaan 1081, \\ Amsterdam $1081 \mathrm{HV}$, Netherlands \\ ${ }^{3}$ ASML Netherlands B.V., De Run 6501, Veldhoven 5504 DR, Netherlands \\ ${ }^{4}$ Department of Applied Physics, Eindhoven University of Technology, Den Dolech 2, Eindhoven 5600 MB, \\ Netherlands
}

(Received 19 September 2019; revised manuscript received 6 December 2019; accepted 15 January 2020; published 13 February 2020)

We experimentally study the morphology of a radially expanding sheet of liquid tin, formed by nanosecond-pulse Nd:YAG laser impact on a spherical microdroplet. Specifically, the sheet thickness profile and its time evolution are captured in detail over a range of laser-pulse energies and for two droplet sizes. Two complementary methods to determine this thickness are employed and shown to be in excellent agreement. All obtained thickness profiles collapse onto a single self-similar curve. Spatial integration of the thickness profiles allows us to determine the volume of the sheet. Remarkably, less than half of the initial amount of tin remains in the sheet under conditions relevant for industrial sources of extreme ultraviolet light, where these thin tin sheets serve as target material. Further analysis shows that the dominant fraction of the mass lost from the sheet during its expansion ends up as fine fragments. We propose that such mass loss can be minimized by producing the sheet targets on the shortest possible time scale. These findings may be particularly valuable for ongoing developments in state-of-the-art nanolithography.

DOI: 10.1103/PhysRevApplied.13.024035

\section{INTRODUCTION}

Tin microdroplets serve as mass-limited targets for highenergy lasers to produce a hot and dense plasma that emits extreme ultraviolet (EUV) light for state-of-the-art nanolithography [1-7]. The generation of EUV light is a two-step process, in which a first laser prepulse deforms the tin droplet into a liquid sheet that is suited to serving as a target for a second, more energetic main laser pulse that produces the EUV light. A detailed understanding of the prepulse-induced deformation is required to optimally produce EUV light with a minimal production of microparticulate "debris." Such debris could limit the lifetime of nearby light-collection optics [4,6-9]. Several recent studies have provided insight into the response of a tin microdroplet to a nanosecond laser pulse in which the propulsion $[10,11]$ accompanies a deformation [1014] of the droplet into a thin sheet. The eventual breakup and fragmentation of the sheet into much smaller droplets

\footnotetext{
*o.versolato@arcnl.nl
}

Published by the American Physical Society under the terms of the Creative Commons Attribution 4.0 International license. Further distribution of this work must maintain attribution to the author(s) and the published article's title, journal citation, and DOI. has also been studied $[9,15]$. The thickness evolution of expanding sheets formed by the impact of millimetersized water drops onto a pillar has been investigated in Refs. [16,17]. Droplet deformation and fragmentation upon impact, be it onto a solid substrate or by a laser pulse, is a topic of particular current interest in fields ranging from epidemiology, governing the transport of pathogens from sneezes and coughs $[17,18]$, through sprays relevant in, e.g., agriculture $[18,19]$, to state-of-the-art nanolithography [7,10-14]. Knowledge of the thickness profile of the thin liquid sheet formed upon impact is crucial for understanding the deformation and complex fragmentation processes involved. However, to date no experimental data are available on the thickness profile of the stretching nanometric liquid metal sheet formed after laser impact. Several theoretical models have been suggested $[13,17,20]$ and applied to tin microdroplets [15] but it has not yet been possible to draw any clear conclusions regarding the thickness profile. Obtaining such information would enable us to estimate how much tin mass is actually retained in the expanding target sheet when it is hit by the main laser pulse in plasma sources of EUV light.

In this work, two complementary methods for determining the sheet thickness profile of laser-impacted tin microdroplets are introduced, compared, and applied under industrially relevant conditions. First, the finite 
transmissivity of the several-tens-of-nanometers-thick stretched liquid-metal sheet is recorded. As the optical constants of tin are well known [21], the transmissivity can be converted to a local thickness $h(r)$ as a function of the radial coordinate $r$, assuming cylindrical symmetry. Second, the Taylor-Culick opening speeds [22] of spontaneously formed holes in the stretching sheet are experimentally measured, which again allows us to obtain $h(r)$. Next, we employ an existing self-similar model [17] of the sheet-thickness evolution and find good agreement between this model and our experiments. Finally, we use the measurement data to determine the fraction of the volume - or, equivalently, mass - that is retained in the target at times relevant for main laser-pulse impact. Channels for mass loss from the sheet are subsequently identified and quantified using existing models $[18,20]$.

\section{EXPERIMENTS AND METHODS}

Our experimental setup is described in detail in Ref. [10]. The most important aspects and relevant upgrades are discussed here. A tin reservoir is situated on top of a vacuum chamber $\left(10^{-7} \mathrm{mbar}\right)$ and is kept at a constant temperature of $260^{\circ} \mathrm{C}$. From the reservoir, a nozzle produces a multikilohertz droplet train of $99.995 \%$-purity tin (with liquid density $\rho=7.0 \mathrm{~g} / \mathrm{cm}^{3}$ and surface tension $\sigma=0.54 \mathrm{~N} / \mathrm{m}$ ) that goes through the center of the chamber. The microdroplets relax to a spherical shape with a diameter set to be either $D_{0}=32$ or $26 \mu \mathrm{m}$ before passing through a horizontal light sheet produced from a helium-neon laser. The resulting scattered light is detected by a photomultiplier tube, the signal from which is frequency down-converted to $10 \mathrm{~Hz}$ to enable triggering of the Nd:YAG laser system. This laser system produces pulses at a wavelength of $1064 \mathrm{~nm}$ and for a time duration of $10 \mathrm{~ns}$ at full width at half maximum (FWHM). The beam is focused to a Gaussian spot (105 $\mu$ m FWHM) onto the droplets in the center of the chamber. By using a half-wave plate and a thin-film polarizer, the beam energy can be adjusted without affecting the spatial beam profile. To maintain cylindrical symmetry, the laser light entering the chamber has circular polarization, produced by employing a quarter-wave plate. The dynamics of the expanding droplets are captured by stroboscopic shadowgraphy imaging systems $[10,11,23]$. These systems are based on two broadband $560 \mathrm{~nm}$ wavelength (with a $12 \mathrm{~nm}$ FWHM bandwidth) 5-ns-long light pulses combined with three long-distance microscopes coupled with CCD cameras. The dye lasers provide backlighting that is rendered both temporally and spatially incoherent to avoid imaging artifacts. The spatial resolution of the imaging systems is approximately $5 \mu \mathrm{m}$. Two microscopes are aligned $\pm 30^{\circ}$ to the Nd:YAG laser beam propagation direction, giving two slightly tilted front-view images. A third microscope is aligned orthogonally to the Nd:YAG laser beam to provide side-view images. The microscopes are equipped with band-pass filters to suppress light emitted by the plasma induced by the laser-pulse ablation. The trigger signals for the two dye lasers are separately produced from two tunable delay generators, which enables us to adjust the time interval between images captured by the two $\pm 30^{\circ}$ microscopes.

\section{A. Sheet thickness from transparency measurement}

Figure 1 presents typical shadowgraph images. The impact of the laser pulse accelerates the droplet to a centerof-mass speed $U$ of order $100 \mathrm{~m} / \mathrm{s}$ and radially deforms it into a thin sheet with a radius $R(t)$ of order $100 \mu \mathrm{m}$ on a time scale of several microseconds. The sheet expansion is axisymmetric, enabling its description in cylindrical coordinates $(r, \theta, z)$. Figures 1(a1.1), 1(a2.1), and 1(a3.1) further show many small fragments created by the destabilization of ligaments that have formed on the edge of the sheet. These ligaments and small droplets extend radially outward in the plane of the sheet. Figures 1(a1.3), 1(a2.3), and 1(a3.3) show corresponding side-view shadowgraph images. These images indicate the presence of a weak curvature of the otherwise smooth liquid sheet that is thus seen to resemble an inverted umbrella. This curvature precludes obtaining the thickness directly from the side views. The curvature, typically with an angle $<2^{\circ}$ over the majority of the sheet, increases near the peripheral edge to $<30^{\circ}$. Similar to Ref. [16], we employ an optical method based on the transmission of light through the sheet to determine the sheet thickness. In our method, however, we probe much thinner sheets. The highly stretched sheet is partially transparent to the backlighting, as is shown with a digitally enhanced contrast in Figs. 1(a1.2), 1(a2.2), and 1(a3.2). For a sheet of material with thickness $h_{m}$ and complex refractive index $n_{m}$ [21], the transmission $T\left(h_{m}\right)$ of a beam through the sheet as a function of thickness is given by [24]

$$
\begin{aligned}
& T\left(h_{m}\right) \\
& \quad=\frac{\left[(1-\mathcal{R})^{2}+4 \mathcal{R} \sin ^{2} \delta\right] e^{-2 \beta h_{m}}}{\left(1-e^{-2 \beta h_{m}} \mathcal{R}\right)^{2}+4 e^{-2 \beta h_{m}} \mathcal{R} \sin ^{2}\left(\delta+\alpha h_{m}\right)},
\end{aligned}
$$

with the reflectance under normal incidence given by $\mathcal{R}=$ $\left|\left(n_{0}-n_{m}\right) /\left(n_{0}+n_{m}\right)\right|^{2}$, where $n_{0}$ is the vacuum refractive index i.e. $n_{0}=1$ and $\delta=\arctan \left[2 k_{0} \beta /\left(\alpha^{2}+\beta^{2}-k_{0}^{2}\right)\right]$, in which $k_{0}$ is the wave number of the incident beam in vacuum. The wave vector inside the material is given by $\alpha=\operatorname{Re}\left[\begin{array}{ll}k_{0} & n_{m}\end{array}\right]$ and $\beta=\operatorname{Im}\left[\begin{array}{ll}k_{0} & n_{m}\end{array}\right]$. Taking the $30^{\circ}$ frontview angle into account, the actual tin-sheet thickness $h=h_{m} \cos 30^{\circ}$. The small incident angle has a negligible influence on the reflectance $\mathcal{R}$.

Equation (1) allows us to estimate the tin-sheet thickness from the CCD pixel values $P_{i j}$ of the shadowgraph images. The local transmission is given by $T_{i j}=\left(P_{i j}-\right.$ 


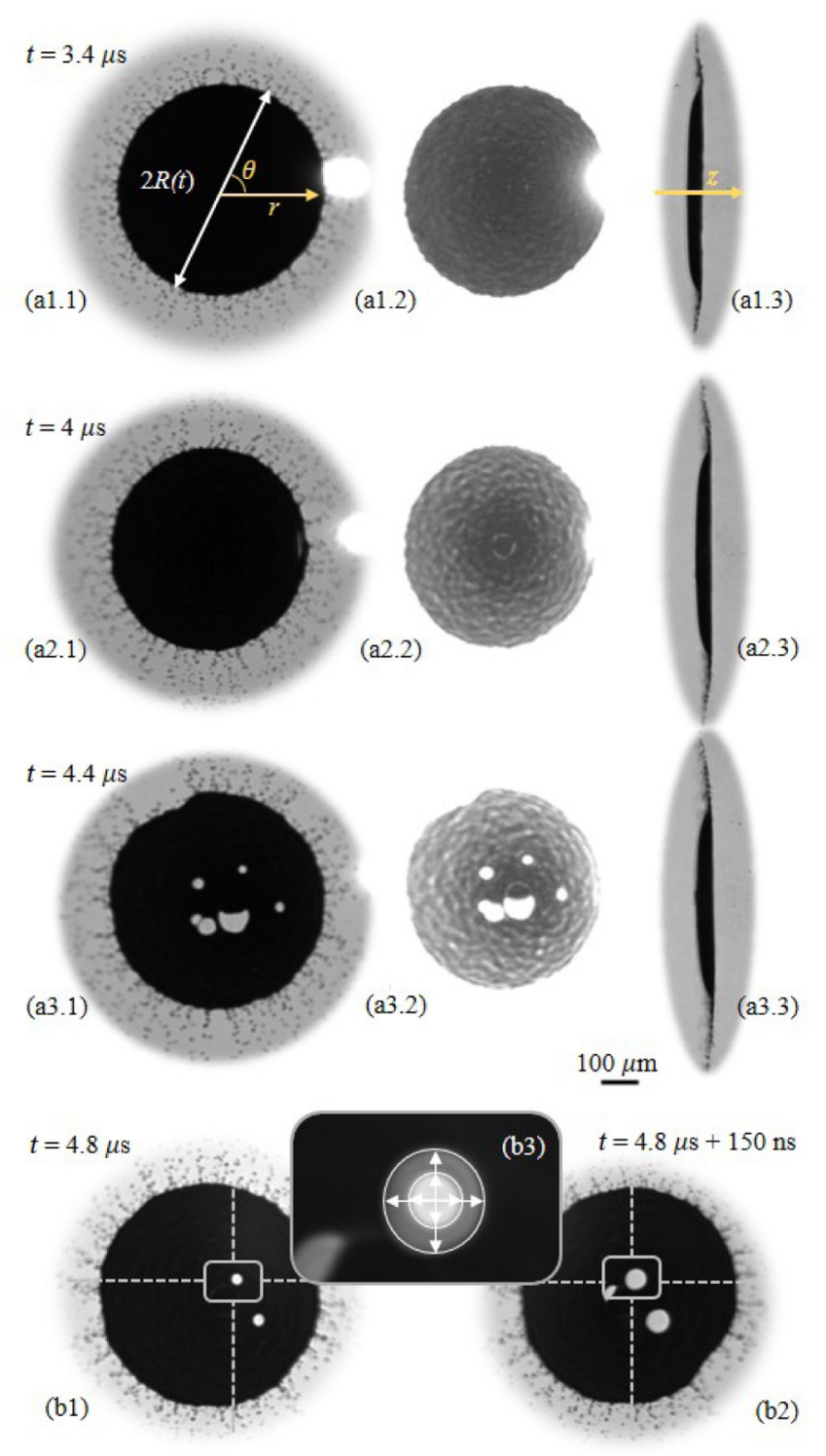

FIG. 1. Shadowgraph images of expanding sheets from tin microdroplets $\left(D_{0}=32 \mu \mathrm{m}\right)$ hit by a nanosecond laser pulse with total energy $E=55 \mathrm{~mJ}$. The droplet accelerates to a velocity $U=160 \mathrm{~m} / \mathrm{s}(\mathrm{We}=5400)$. (a1.1),(a2.1),(a3.1) Front views of liquid sheets at time delay $t$ (laser impacts at $t=0$ ). The images are stretched along the horizontal axis to correct for the $30^{\circ}$ observation angle. The bright spots in the images are from plasma emission. (a1.2),(a2.2),(a3.2) The same images with a digitally modified contrast. (a1.3),(a2.3),(a3.3) Corresponding side-view images indicate a weak curvature (laser impacts from the left, propelling the droplet to the right). In (a1.1) and (a1.3), the cylindrical coordinate system $(r, \theta, z)$ with its origin at the center of the sheet is depicted. At later times, holes appear, cf. (a3), (b1), and (b2). The overlay of a hole (see the main text) is highlighted by a white box in both (b1) and (b2). The arrows in inset (b3) indicate the receding edge of the hole.

$\left.P_{0}\right) /\left(P_{b, i j}-P_{0}\right)$, where $i$ and $j$ are the pixel indices. Backlighting values $P_{b, i j}$ are obtained from shadowgraphs where no droplets are present in the region of interest. The values for $P_{i j}, P_{b, i j}$, and $P_{0}$ are discrete and have a limited, 8 -bit dynamic range. Therefore, averaging over sufficient (typically 20) images is required to obtain an accurate mean value to represent the actual smooth transmission profile. Parameter $P_{0}$ characterizes a common background offset value that could originate from, e.g., dark noise or imaging glare. This parameter is experimentally established using thick fully opaque tin sheets in an early stage of deformation or particularly thick features within the sheets. The values of $P_{0}$ depend on the intensity of the backlighting, the particulars of the optical alignment, and the intensity of the plasma emission. These influences are taken into account by individually assessing $P_{0}$ for each shadowgraph image. We apply the transparency method for sheets with $h \lesssim 50 \mathrm{~nm}$ and obtain spatial thickness profiles $h(r)$ spanning the full range $0 \leq r \leq R(t)$.

\section{B. Sheet thickness from hole opening speed}

In Figs. 1(a1.2), 1(a2.2), and 1(a3.2), we observe ripples. These ripples are the signature of a fluid-dynamic instability that will eventually puncture the sheet and cause the formation of holes $[15,25]$. On uniform sheets, these holes open at a constant Taylor-Culick (TC) speed [22],

$$
u_{t c}=\sqrt{\frac{2 \sigma}{\rho h}},
$$

which expresses a balance between surface tension and inertia. After aligning the corresponding oblique $\left( \pm 30^{\circ}\right)$ front-view images with the centers of each pair of holes, we determine the distance traveled by the hole boundary [see Fig. 1(b3)]. From these distances and the time interval between the two images, we obtain the local TC speed and from Eq. (2) the local sheet thickness. Figures 1(b1), 1(b2), and 1(b3) show typical examples of the results of such alignment for a hole. First, an expanding sheet is captured by one camera at $t=4.8 \mu \mathrm{s}$ [see Fig. 1(b1)]. Then, $150 \mathrm{~ns}$ later, the same target is recorded by the other frontview camera [see Fig. 1(b2)]. We align Figs. 1(b1) and 1(b2) with the center of this hole in Fig. 1(b3), where the two images are overlaid as visualized using different grayscale values. We clearly observe the increase in the size of the hole with time. The distance traveled by the hole boundary is determined by tracing four points defined by the intersection of an orthogonal cross with the boundary. The local thickness is obtained for each point individually; the radial coordinate is provided by a correction procedure that takes into account the finite curvature close to the edge of the sheets (cf. the side views in Fig 1), which causes an apparent horizontal shift in the position of the hole in the two front-view shadowgraphs. With holes appearing nearly randomly over the sheet surface, an almost complete thickness profile $h(r)$ can be constructed by processing sufficient stroboscopic images taken at the same time delay. 
Holes appearing near the edge of the sheet cannot be used in the TC method due to significant nonuniformity of the local thickness profile [15]. In addition, the edge curvature distorts the hole overlay close to the sheet circumference. The TC method is only applicable within approximately $1 \mu \mathrm{s}$ of the onset of hole formation $\tau^{*}$. At later times, many holes quickly appear and obstruct the analysis. Time $\tau^{*}$ has previously been observed to decrease with the Weber number $\mathrm{We}=\rho R_{0} U^{2} / \sigma$ as $\tau^{*} / \tau_{c} \sim \mathrm{We}^{-1}$ [15]. Thus, with an increasing Weber number, the thickness is probed by the TC method at earlier times. Here, the Weber number is defined with the initial droplet radius $R_{0}=D_{0} / 2$ and the center-of-mass speed $U$. With the laser-pulse energy ranging from 40 to $200 \mathrm{~mJ}, U$ ranges from 100 to $300 \mathrm{~m} / \mathrm{s}$, yielding $\mathrm{We}=2000-20000$ (see the Appendix). The capillary times characterizing the capillary-sheet retraction $\tau_{c}=\sqrt{\rho R_{0}^{3} / \sigma}$ are $\tau_{c}=7.3$ and $5.3 \mu$ s for droplet sizes $D_{0}=32$ and $26 \mu \mathrm{m}$, respectively.

\section{RESULTS}

\section{A. Thickness profile}

Figure 2 presents a selection of the measurement results for the sheet thickness obtained from the two methods: (a) transmission (with droplet sizes $D_{0}=32,26 \mu \mathrm{m}$ ) and (b) TC speed (with $D_{0}=32 \mu \mathrm{m}$ ). A comparison of the two methods in the parameter range in which both methods are applicable is presented in Fig. 2(c). Figure 2(a) shows that after impact from a laser pulse, the droplet deforms into a sheet with a thickness of several tens of nanometers. The profiles have a slender spatial gradient, where $h$ decreases with $r$. The thickness $h$ generally decreases with time, as becomes clear comparing the $55-\mathrm{mJ}$ pulse cases at different time delays. These features are consistent with the TC results presented in Fig. 2(b), where the reduction of the thickness with time is seen by comparing the two $50-\mathrm{mJ}$ cases. Laser pulses with higher energies deform the droplet faster: to thin the droplet to the observed thickness, it takes $4.3 \mu \mathrm{s}$ for a $50-\mathrm{mJ}$ laser pulse, while a shorter time of $2.5 \mu \mathrm{s}$ is required to reach a similar thickness for a more energetic pulse of $120 \mathrm{~mJ}$. All cases shown present a profile that, with time, decreases both in thickness and in radial dimension. The reduction of the sheet radius occurs on the time scale of capillary retraction of the sheet [20]. Figure 2(a) also shows the data from the smaller droplet size $D_{0}=26 \mu \mathrm{m}$, demonstrating that the thickness evolution is consistent with that of the larger droplet: $h$ reduces both in $t$ and $r$ and decreases faster with more energetic laser pulses.

The comparison of the two methods presented in Fig. 2(c) shows excellent agreement. This agreement validates our two methods of measurement. Some discrepancies appear in a small region of size approximately $R_{0}$ close to the origin, where both the transmission and the TC

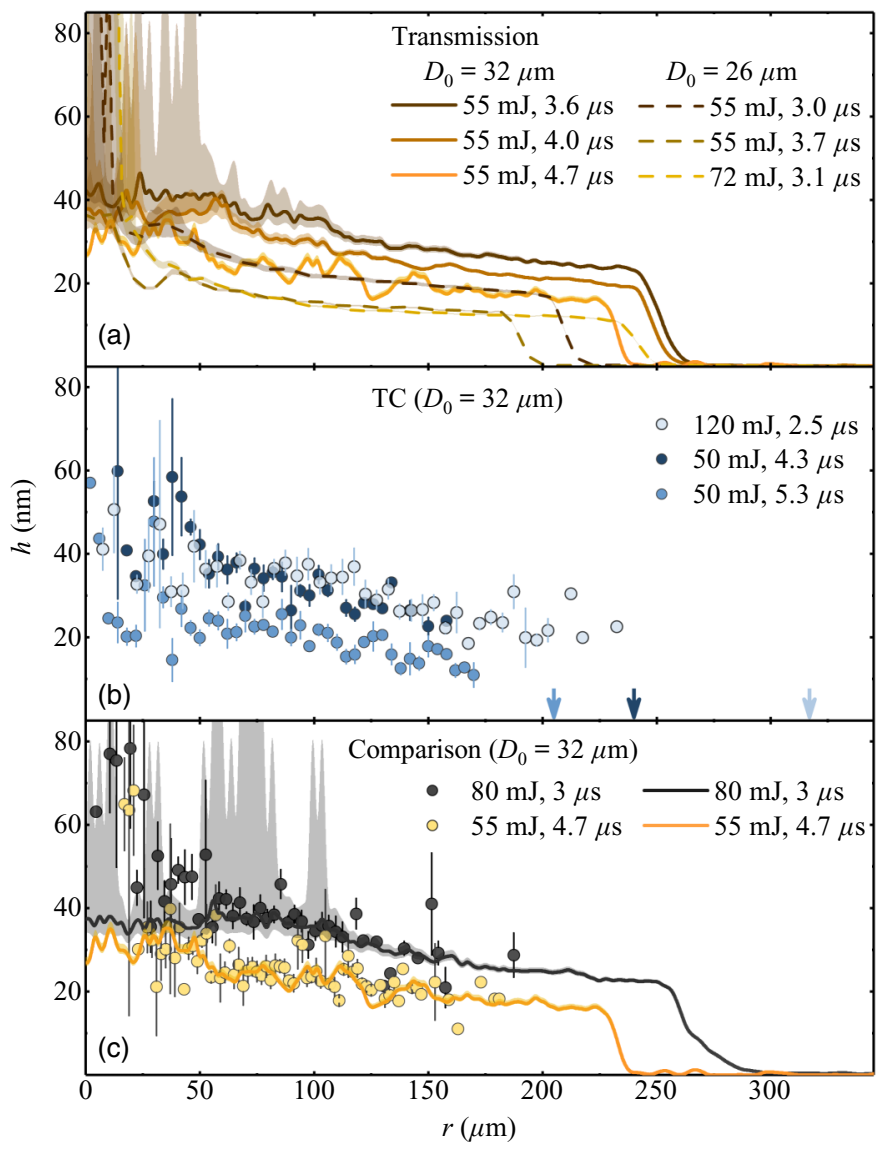

FIG. 2. The sheet thickness as a function of the radial coordinate. (a) Results from the transmission method. Data from $D_{0}=32$ and $26 \mu \mathrm{m}$ droplets are depicted by solid and dashed lines, respectively. The shaded areas following the data curves indicate the typical systematic uncertainties obtained by varying $P_{0} \pm 3 \%$. (b) Results from the TC method with $D_{0}=32 \mu \mathrm{m}$. Arrows positioned on the $x$ axis represent the sheet radius. The uncertainty error bars are based on the standard deviation of binned data. (c) A comparison of the two methods.

methods hint at the presence of a thicker feature. Visual inspection of Figs. 1(a2.2) and 1(a3.2) confirms the presence of such thick disklike features near the center of the sheet bounded by a much thinner halo. The stochastic nature of this feature leads to a locally increased standard deviation error on the binned data, e.g., as shown in Fig. 2. These features may be caused by jetting and bursting, phenomena that could originate from laser-induced cavitation inside the liquid [14,15,23]. The amount of mass contained in these features is expected to be small [15].

The dominant fraction of the data presented in Fig. 2 shows smooth thickness profiles $h(r, t)$ that decrease with both time and the radial coordinate. Under all experimental conditions and over all time ranges studied, we observe that the sheet profiles share remarkably similar shapes. 


\section{B. Model of the self-similar thickness profile}

Following the work by Wang et al. [17] for sheet expansion upon droplet impact on a pillar, we use a self-similar solution to describe the sheet-thickness evolution. In this slender-slope model, the flow $u_{r}(r, t)$ in the expanding sheet is assumed to be radially outward. Furthermore, the flow is considered to be inviscid (the Reynolds number is of order $10^{3}$ ) and curvature-induced radial pressure gradients are neglected. The flow in the sheet is then given by the axisymmetric Euler equation

$$
\frac{\partial u_{r}}{\partial t}+u_{r} \frac{\partial u_{r}}{\partial r}=0 .
$$

Equation (3) suggests that a flow field $u_{r}(r, t)=r / t$ is established on the time scale of sheet expansion, i.e., at times $t>D_{0} / U \sim 100 \mathrm{~ns}$. Completing Eq. (3) by the massconservation equation for an axisymmetric sheet, one finds the (nondimensionalized) governing equation for the sheet expansion [17]

$$
t^{\star} \frac{\partial h^{\star}}{\partial t^{\star}}+r^{\star} \frac{\partial h^{\star}}{\partial r^{\star}}+2 h^{\star}=0,
$$

where $r^{\star}=r / D_{0}, h^{\star}=h / D_{0}$, and $t^{\star}=U t / D_{0}$. The similarity solution to Eq. (4) is of the form [17]

$$
h^{\star} t^{\star 2}=f\left(\frac{r^{\star}}{t^{\star}}\right) \text {. }
$$

Equation (5) predicts that all experimental data $h(r, t$, $\left.U, D_{0}\right)$ can be collapsed onto a universal curve given by $y=f(x)$ with similarity variables $x=r^{\star} / t^{\star}, y=$ $h^{\star} t^{\star 2}$. Figure 3(a) demonstrates the collapse upon rescaling in terms of the similarity variables for the all obtained data - which, for clarity, are subdivided into the transmission-method results in Fig. 3(b) and the TCmethod results in Fig. 3(c). Without exception, all data collapse onto a single master curve.

For the similarity profile $f$ in Eq. (5), several theoretical and empirical conjectures have been proposed for droplet impact on a solid [17,26-28]. Inspired by Wang et al. [17], we formulate $f(x)$ for the tin-sheet expansion upon laserpulse impact as

$$
f(x)=\frac{1}{a_{0}+a_{1} x+a_{2} x^{2}},
$$

with its associated dimensional thickness profile

$$
h\left(r, t, U, D_{0}\right)=\frac{D_{0}^{3}}{a_{0} U^{2} t^{2}+a_{1} U t r+a_{2} r^{2}} .
$$

The result of the fit of Eq. (6) to the full concatenated data yields $a_{0}=1.65(2), a_{1}=6.9(3)$ and $a_{2}=-2.4(8)$, with the number in brackets indicating the one-standarddeviation uncertainty. Data points within the central disk feature (typically of size approximately $R_{0}$ ), as determined by individual inspection of the shadowgram images, are excluded from the fit.
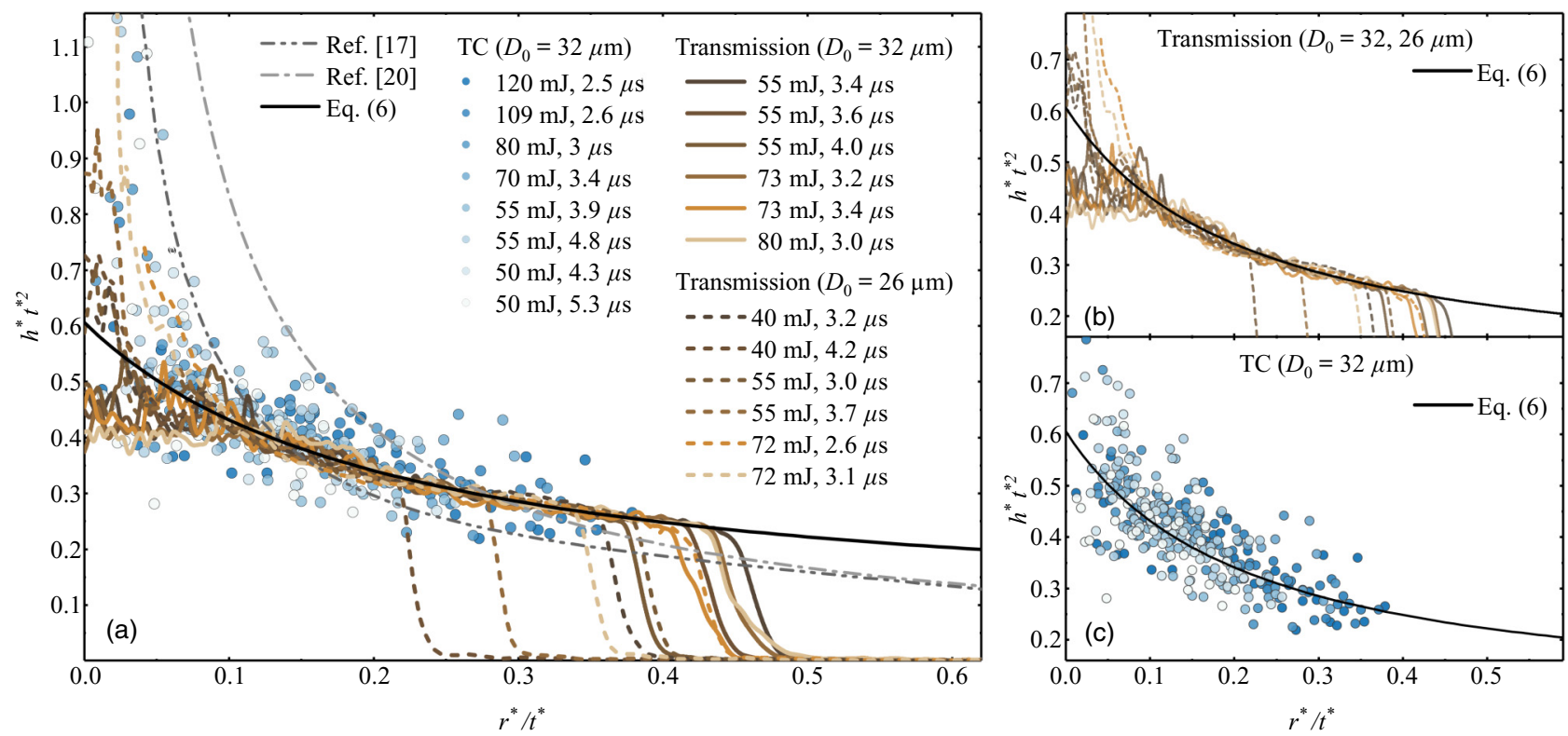

FIG. 3. (a) The sheet-thickness data represented in terms of the similarity variables $r^{\star} / t^{\star}$ and $h^{\star} t^{\star 2}$ (see the main text). The transmission data from $D_{0}=32$ and $26 \mu \mathrm{m}$ are depicted by colored solid and dashed lines, respectively; TC data from $D_{0}=32 \mu \mathrm{m}$ are represented by scatter points. The corresponding pulse energy and time delay are indicated in the legend. The fit result of Eq. (6) to the full concatenated data range is depicted by a black solid line. Other models from Refs. [17,20] are also shown (see the legend). (b) A plot of the transmission data only. (c) A plot of the TC data only. 
Equations (6) and (7) are of the same form as the ones in Ref. [17] but differ in the coefficients of the polynomial. Indeed, using the semiempirical coefficients $a_{1}=23.2$, $a_{2}=-38.4$, and a cubic term $a_{3}=34.2$ from Ref. [17], we find an overestimation of the thickness near the center of the sheet and an underestimation of it closer to the edge, as shown in Fig. 3. These discrepancies reflect the different physical origins of the droplet deformation in the two cases: in our experiments it is the laser-produced plasma pressure that causes the deformation of the droplet into a sheet $[10,11,23]$, while in the experiments by Ref. [17] it is the impact onto a solid surface. This difference affects the early-time deformation of the droplet; in particular, near the impact center. Therefore, we use an additional term $a_{0}$ to account for the different boundary condition at $r=0$ and obtain different numerical values for $a_{1}, a_{2}$. Furthermore, we remove the cubic term that describes the early-time $\left(t \ll D_{0} / U\right)$ deformation dynamics [17] and does not impact the current late-time sheet expansion.

The collapsed experimental data for our tin droplets are seen to be excellently reproduced by Eq. (7). Hence, Eq. (7) provides a useful tool for predicting the thickness profile of expanding sheets for all droplet sizes and laserimpact parameters at times relevant for nanolithography, i.e., at times $t \gg D_{0} / U$ when significant target expansion has occurred.

\section{Mass distribution during sheet expansion}

As shown in Fig. 2, the stretched sheet is several tens of nanometers in thickness. This thickness is a factor of one half to one quarter of what one would estimate from global mass conservation by assuming a uniform sheet of thickness $h=4 R_{0}^{3} /\left[3 R(t)^{2}\right]$. Additionally, at the time scales probed in this study, the thickness and the radius of the sheet decrease simultaneously with time (see Fig. 2). These findings suggest that a substantial amount of tin is removed from the sheet during its expansion.

To better understand the temporal and spatial distribution of the tin mass, we again revisit studies of droplet impact on a pillar $[16-18,20,26]$ or on a solid substrate $[27,29]$. In these studies, the Weber number $\mathrm{We}=$ $\rho U^{2} R_{0} / \sigma$ is the pertinent parameter to describe the expansion dynamics. To facilitate a direct comparison with that body of available literature, we group our data by the Weber number hereafter, using the drop's center-of-mass speed $U$ (see the Appendix).

A doughnut-shaped rim formed on the bounding edge of the expanding sheet has been universally reported. This rim progressively collects mass, ever increasing its volume $V_{\text {rim }}$. This rim cannot be seen separately in Fig. 1 as it has a diameter (see below) well below our resolution limit. It destabilizes due to capillary instabilities and forms ligaments that move radially outward and break into a fine spray of droplets $[15,20]$, which comprise a volume
$V_{\text {fragment }}$. These ligaments and fragment droplets are clearly visible in Fig. 1. Thus, in addition to the volume in the sheet $V_{\text {sheet }}$, we ultimately interpret the tin distribution as

$$
V_{0}=V_{\text {sheet }}+V_{\text {rim }}+V_{\text {fragment }}
$$

during the sheet expansion, which we will now analyze. In the following, given the constraints on the transmission and TC methods, we consider only the case of mostly intact sheets, i.e., those where no significant hole formation has occurred yet. Further experimental progress toward better visibility of fragment droplets may in the future enable a direct quantification of the fragment volume.
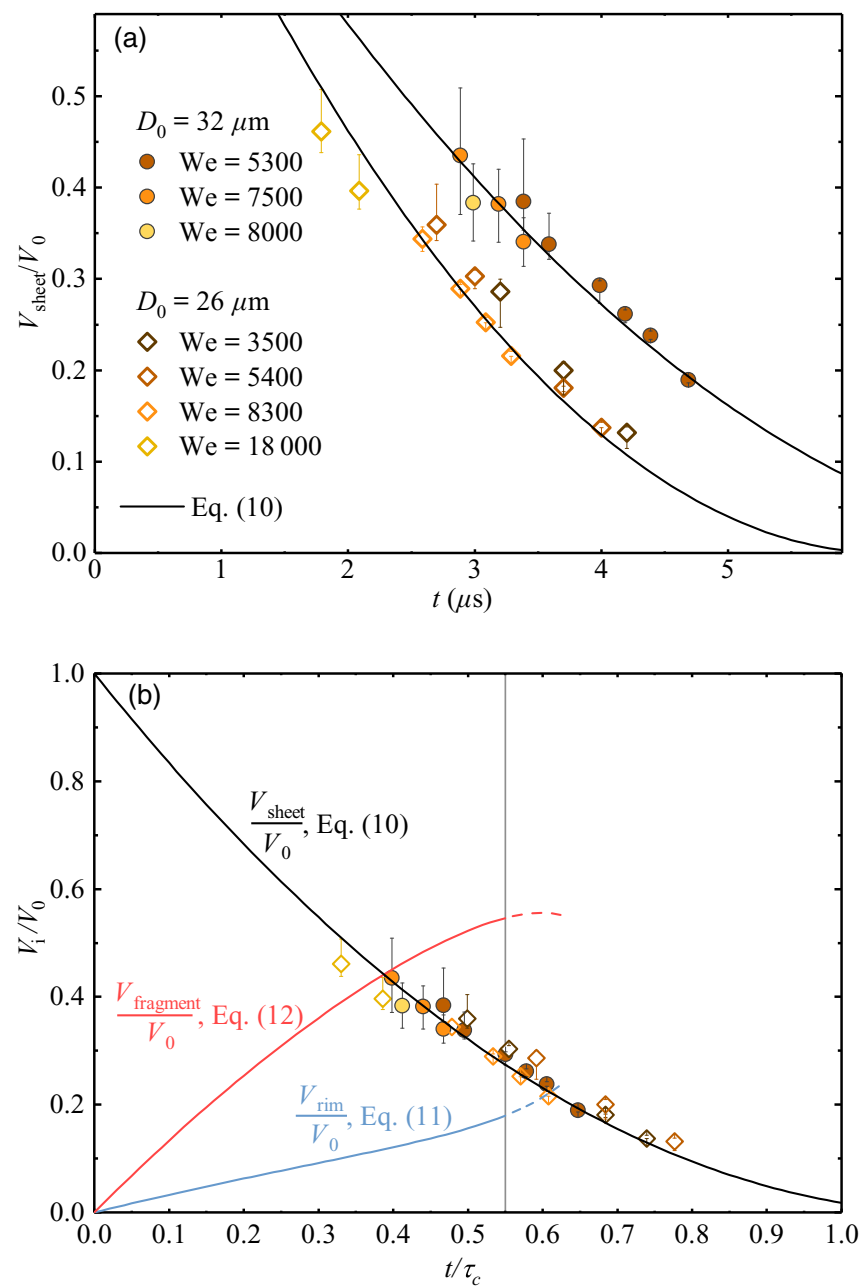

FIG. 4. (a) The volume ratio of the sheet to that of the initial droplet $V_{\text {sheet }} / V_{0}$ as a function of time for droplet sizes $D_{0}=32,26 \mu \mathrm{m}$. The Weber numbers for the various data sets are indicated in the legend. (b) The volume ratio $V_{\mathrm{i}} / V_{0}$ as a function of the nondimensional time $t / \tau_{c}$. Equation (10) for $V_{\text {sheet }} / V_{0}$ is represented by black solid lines in both panels. The volume ratios $V_{\text {rim }} / V_{0}$ from Eq. (11) and $V_{\text {fragment }} / V_{0}$ from Eq. (12) are depicted as blue and red lines, respectively. The vertical line marks $t / \tau_{c} \approx 0.55$ (see the main text). 


\section{Volume of the sheet}

Figure 4(a) presents the volume ratio of the sheet to the initial droplet $V_{\text {sheet }} / V_{0}$ as a function of time with Weber numbers between 3500 and 18000 . The volume $V_{\text {sheet }}$ is obtained by integrating over the transmission data in rings contributing $\delta V_{\text {sheet }}=2 \pi r h \delta r$. TC data are not used here, as they do not provide a description of $h(r)$ over the full domain of $r$. Over time, the sheet continuously loses liquid: about $2 \mu$ s after the laser-pulse impact, less than half the initial amount of tin is still in the sheet. For the smaller droplet $\left(D_{0}=26 \mu \mathrm{m}\right)$, the fraction $V_{\text {sheet }} / V_{0}$ is smaller than that of the larger $\left(D_{0}=32 \mu \mathrm{m}\right)$ droplet at equal times.

To derive a theoretical expression for the sheet volume, we require not only an expression for the time-dependent sheet thickness $h(r, t)$ but also for the sheet radius $R(t)$. To this end, we employ the model developed by Villermaux et al. [20] for pillar impact, in which the sheet radius is given by

$$
\frac{R(t)-R_{0}}{R_{0}}=\sqrt{\mathrm{We}} \frac{t}{\tau_{c}}\left(1-\frac{t}{\tau_{c}} \frac{\sqrt{3}}{2}\right)^{2} .
$$

This expression has previously been found to agree well with tin-droplet expansion after laser-pulse impact $[10,15]$. The thickness profile that corresponds to Eq. (9) is given by $f(x)=1 /(12 x)$ in Ref. [20], which is consistent with Eq. (6) taking $a_{1}=12$ and $a_{0}=a_{2}=0$. This thickness profile is in quite reasonable agreement with our data, given the fact that the model does not contain any free fit parameter (see Fig. 3). Using Eq. (9) together with this thickness profile, we obtain, for the sheet volume,

$$
\frac{V_{\text {sheet }}}{V_{0}}=\frac{1}{V_{0}} \int_{R_{0}}^{R(t)} 2 \pi r h(r, t) d r=\left(1-\frac{\sqrt{3}}{2} \frac{t}{\tau_{c}}\right)^{2} .
$$

This result shows that the sheet volume is independent of the Weber number and, furthermore, is solely parametrized by the nondimensional time $t / \tau_{c}$. Note that the integration in Eq. (10) starts from $r=R_{0}$, as the original model assumes $u_{r}=0$ at the pillar edge, with the sheet remaining attached to the pillar while it expands [20]. To legitimize the comparison of the experiment with this model, the integration of the experimental data is performed over the same interval. The omitted center region (i.e., $r \in\left[0, R_{0}\right]$ ) may further contribute to the total volume with the disk feature contained in it (see Sec. III 3). Taking as inputs the values of $\tau_{c}$ (i.e., 7.3 and $5.3 \mu$ s for droplet sizes $D_{0}=32$ and $26 \mu \mathrm{m}$, respectively), we find that Eq. (10) is able to predict the volume ratio with a high degree of accuracy [see Fig. 4(a)]. Figure 4(b) shows the same data but now as a function of $t / \tau_{c}$, along with Eq. (10). All data collapse together onto the universal curve given by Eq. (10), which demonstrates that $t / \tau_{c}$ is indeed the only relevant parameter for describing the volume fraction of the sheet.

\section{Volume of the rim and fragmentation}

Having experimentally concluded that the sheet contains less than half the total available mass in the time domain investigated, we turn to a theoretical analysis of the channels of mass loss from the sheet. As discussed above, a bounding rim on the edge of the sheet develops during sheet expansion, which has a volume $V_{\text {rim }}=$ $\pi^{2} R(t) b^{2} / 2$, where $b$ is the rim diameter. Recently, it has been shown that the rim diameter $b$ of an expanding liquid sheet that undergoes unsteady fragmentation is universally governed by a local instantaneous Bond number Bo $\equiv-\ddot{R}(t) \rho b^{2} / \sigma=1$ [18]. We again refer to Eq. (9) for the expansion radius and the associated instantaneous deceleration $\ddot{R}(t)$ and obtain, upon substitution into $\mathrm{Bo}=1$,

$$
\begin{aligned}
\frac{V_{\mathrm{rim}}}{V_{0}}= & \frac{3 \pi}{16} \\
& \times\left[\frac{3\left(t / \tau_{c}\right)^{3}-4 \sqrt{3}\left(t / \tau_{c}\right)^{2}+4\left(t / \tau_{c}\right)+4\left(R_{0} / U \tau_{c}\right)}{4 \sqrt{3}-9\left(t / \tau_{c}\right)}\right] .
\end{aligned}
$$

Since $R_{0} /\left(U \tau_{c}\right)=\mathrm{We}^{-1 / 2} \ll 1$ for all cases studied here, this term can be neglected. Equation (11) can thus be approximated as a function of just $t / \tau_{c}$, shown as the blue line in Fig. 4(b). The Bond-number criterion can be used to determine the rim thickness as long as the rim undergoes a sufficiently large acceleration of $(-\ddot{R}) \sim R_{\max } / \tau_{c}^{2} \sim U / \tau_{c}$ [18]. As in our experiments the rim acceleration decreases with time, we apply Eq. (11) up to the moment at which $(-\ddot{R}) \approx U / \tau_{c}$, from which we obtain, using Eq. (9), $t / \tau_{c} \approx$ 0.55 . This limiting time is indicated in Fig. 4(b) by the black vertical line.

Clearly, the relatively slow monotonic increase in $V_{\text {rim }}$ alone cannot account for the fast decrease in $V_{\text {sheet }}$. This observation suggests that the majority of the mass is lost through the remaining channel of fragmentation $V_{\text {fragment }}$. We express this volume by the complementary fraction

$$
\frac{V_{\text {fragment }}}{V_{0}}=1-\frac{V_{\text {sheet }}}{V_{0}}-\frac{V_{\text {rim }}}{V_{0}},
$$

which is represented by a red line in Fig. 4(b). We note that in contrast to $V_{\text {sheet }}(t)$ and $V_{\text {rim }}(t)$, which represent instantaneous quantities, $V_{\text {fragment }}(t)$ is a cumulative volume representing the total volume of fragments formed up to that point in time. The models presented in Fig. 4 are valid in both expanding and contraction phases of the laser-impacted sheet when the combined requirements of (i) sufficient deformation $\left(t>D_{0} / U\right)$ and (ii) sufficient rim deceleration $\left(-\ddot{R}>U / \tau_{c}\right)$ are met. 


\section{Further contributions to the tin mass distribution}

Besides the contributions $V_{\text {sheet }}, V_{\text {rim }}$, and $V_{\text {fragment }}$, several other weaker channels of mass loss can be identified.

First, mass is lost due to plasma formation upon the impact of the laser pulse onto the droplet. Through momentum conservation, it is this accelerated mass that sets the droplet into motion [10]. Plasma formation may also lead to small splashing events producing some microparticulate debris. Full radiation-hydrodynamics simulations have, however, shown that these mass-loss channels only contribute a few percent [11].

Second, there is the disk feature as discussed in Sec. III A. A direct estimate of the mass contained in this feature can be obtained by considering the residue of the disk after its complete separation from the sheet and its subsequent contraction. Side- and front-view shadowgraphs, where available, enable a volume estimate. A first such investigation, even though limited by the optical resolution, indicates that the mass fraction may be as large as $10 \%$ in certain cases. The size of this contribution and its origins thus warrant further research. Regardless, this disk contribution is relatively minor and will not affect the main conclusions of our studies.

\section{CONCLUSION}

We experimentally study the morphology of a radially expanding sheet of liquid tin formed upon nanosecondpulse laser impact on a spherical microdroplet under conditions relevant for EUV lithography. The thickness profile of the several-tens-of-nanometers-thick tin sheet and its time evolution is captured over a range of Nd:YAG laserpulse energies and two droplet diameters, $D_{0}=26 \mu \mathrm{m}$ and $D_{0}=32 \mu \mathrm{m}$, by employing two complementary methods. First, the transmissivity of the stretching liquid-metal sheet is recorded and used to determine the local thickness. Second, the Taylor-Culick opening speeds of spontaneously formed holes in the stretching sheet are experimentally determined, which allows us to obtain the thickness profile through an independent method. The two methods are shown to be in excellent agreement.

We show that all the sheet thicknesses measured collapse onto a single curve that is well described by the similarity solution proposed in Ref. [17]. Spatial integration of the thickness profiles obtained experimentally provides the volume of the sheet. Remarkably, less than half of the initial droplet volume remains in the sheet under conditions relevant for nanolithography.

Our further theoretical analysis indicates that the dominant fraction of the mass lost from the sheet during its expansion ends up as fragmented droplets. This fragment debris may lead to coating of nearby optic elements and hence may reduce the lifetime of industrial apparatus. To minimize the amount of fragment debris, it is best that the main laser pulse that creates the plasma-emitting EUV light follows the prepulse with the smallest possible time delay. To enable the tin sheet to attain a sufficient size at this short time delay, an energetic prepulse is required to give it the required high expansion speed. These changes would enable optimization of the amount of tin contained in the sheet while minimizing the volume contributing to microparticulate debris in industrial sources of EUV light.

\section{ACKNOWLEDGMENTS}

We thank Professor P. Planken for useful discussions on the transmission of light through thin metal layers. This work has been carried out at the Advanced Research Center for Nanolithography (ARCNL), a public-private partnership of the University of Amsterdam (UvA), the Vrije Universiteit Amsterdam (VU), the Netherlands Organisation for Scientific Research (NWO), and the semiconductor equipment manufacturer ASML. This project has received funding from the European Research Council (ERC) through Starting Grant No. 802648 and is part of the Vidi research program with Project No. 15697, which is financed by the NWO.

\section{APPENDIX: SCALING OF THE WEBER NUMBER WITH PULSE ENERGY}

The dynamics of droplet propulsion by laser-pulse impact have been detailed in Refs. [10,11]. In these works, a scaling law of $U \sim E_{\text {od }}^{0.6}$ has been proposed that relates the center-of-mass speed $U$ to the energy on the droplet, $E_{\text {od }}$, defined as the fraction of the incident pulse energy $E$ that impinges onto the droplet. The Weber number We $=$ $\rho U^{2} R_{0} / \sigma$ should then follow We $\sim E_{\text {od }}^{1.2}$. Figure 5 presents the scaling of the Weber number with the energy on the droplet for three different droplet sizes. It is shown that the data are indeed in excellent agreement with the scaling law. Furthermore, the prefactors are consistent with the observations in Ref. [11], where the prefactor is predicted to be proportional to $R_{0}^{-2.2}$. Accordingly, the Weber numbers from different droplet sizes should follow the same trend when they are appropriately scaled as

$$
\widetilde{\mathrm{We}}=\mathrm{We}\left(\frac{D_{0}}{45 \mu \mathrm{m}}\right)^{3.4}
$$

taking the droplet size in Ref. [11] $\left(D_{0}=45 \mu \mathrm{m}\right)$ as a reference and also the prefactor from Ref. [11]. The inset in Fig. 5 shows that data from different droplet sizes collapse perfectly onto a single curve. These results enable the direct prediction of the Weber number from the laserpulse energy impacting on the droplet over a wide range of experimental parameters. 


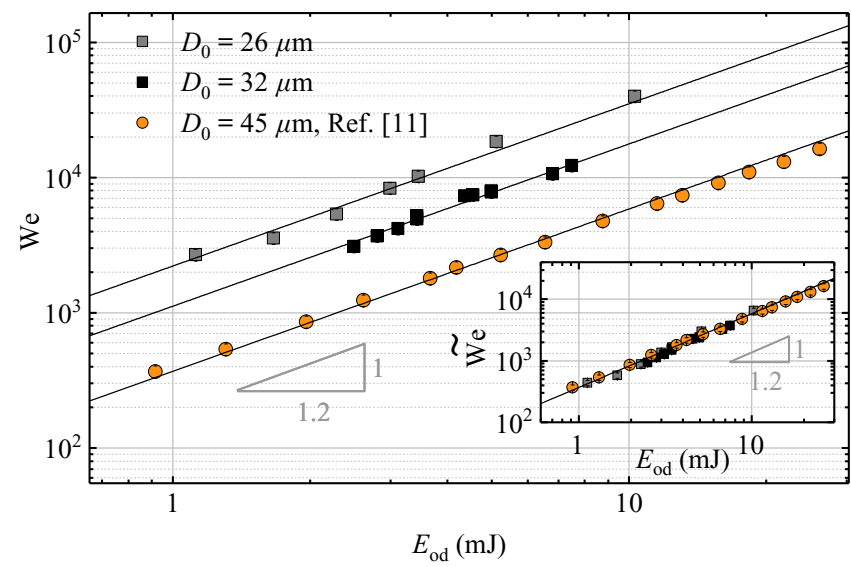

FIG. 5. A double-logarithmic plot of the Weber number We as a function of the energy on the droplet $E_{\text {od }}$ for the two studied droplet sizes. Individual fit results of the scaling law We $\sim E_{\text {od }}^{1.2}$ for different droplet sizes are shown as black lines. Data reproduced from Ref. [11] for a larger droplet diameter $\left(D_{0}=45 \mu \mathrm{m}\right)$ but for a similar beam size (100 $\mu \mathrm{m}$ FWHM) are also depicted, including the original scaling law. The inset shows the rescaled Weber number $\widetilde{W e}$ as a function of $E_{\text {od }}$. The scaling law $\widetilde{\mathrm{We}} \sim$ $E_{\text {od }}^{1.2}$, with its prefactor taken from Ref. [11], is shown by a black line (see the main text).

[1] Y. Tao, M. S. Tillack, K. L. Sequoia, R. A. Burdt, S. Yuspeh, and F. Najmabadi, Efficient $13.5 \mathrm{~nm}$ extreme ultraviolet emission from $\mathrm{Sn}$ plasma irradiated by a long $\mathrm{CO}_{2}$ laser pulse, Appl. Phys. Lett. 92, 251501 (2008).

[2] J. Benschop, V. Banine, S. Lok, and E. Loopstra, Extreme ultraviolet lithography: Status and prospects, J. Vac. Sci. Technol. B 26, 2204 (2008).

[3] S. Fujioka, M. Shimomura, Y. Shimada, S. Maeda, H. Sakaguchi, Y. Nakai, T. Aota, H. Nishimura, N. Ozaki, A. Sunahara, K. Nishihara, N. Miyanaga, Y. Izawa, and K. Mima, Pure-tin microdroplets irradiated with double laser pulses for efficient and minimum-mass extreme-ultraviolet light source production, Appl. Phys. Lett. 92, 241502 (2008).

[4] V. Y. Banine, K. N. Koshelev, and G. H. P. M. Swinkels, Physical processes in EUV sources for microlithography, J. Phys. D 44, 253001 (2011).

[5] I. Fomenkov, in 2017 International Workshop on EUV and Soft X-Ray Sources (Dublin, 2017).

[6] I. Fomenkov et al., Light sources for high-volume manufacturing EUV lithography: Technology, performance, and power scaling, Adv. Opt. Technol. 6, 173 (2017).

[7] O. O. Versolato, Physics of laser-driven tin plasma sources of EUV radiation for nanolithography, Plasma Sources Sci. Technol. 28, 083001 (2019).

[8] R. W. Coons, S. S. Harilal, D. Campos, and A. Hassanein, Analysis of atomic and ion debris features of laserproduced Sn and Li plasmas, J. Appl. Phys. 108, 063306 (2010).

[9] D. Hudgins, N. Gambino, B. Rollinger, and R. Abhari, Neutral cluster debris dynamics in droplet-based laser-produced plasma sources, J. Phys. D 49, 185205 (2016).

[10] D. Kurilovich, A. L. Klein, F. Torretti, A. Lassise, R. Hoekstra, W. Ubachs, H. Gelderblom, and O. O. Versolato, Plasma Propulsion of a Metallic Microdroplet and its Deformation upon Laser Impact, Phys. Rev. Appl. 6, 014018 (2016).

[11] D. Kurilovich, M. M. Basko, D. A. Kim, F. Torretti, R. Schupp, J. C. Visschers, J. Scheers, R. Hoekstra, W. Ubachs, and O. O. Versolato, Power-law scaling of plasma pressure on laser-ablated tin microdroplets, Phys. Plasmas 25, 012709 (2018).

[12] A. L. Klein, W. Bouwhuis, C. W. Visser, H. Lhuissier, C. Sun, J. H. Snoeijer, E. Villermaux, D. Lohse, and H. Gelderblom, Drop Shaping by Laser-Pulse Impact, Phys. Rev. Appl. 3, 044018 (2015).

[13] H. Gelderblom, H. Lhuissier, A. L. Klein, W. Bouwhuis, D. Lohse, E. Villermaux, and J. H. Snoeijer, Drop deformation by laser-pulse impact, J. Fluid Mech. 794, 676 (2016).

[14] S. A. Reijers, J. H. Snoeijer, and H. Gelderblom, Droplet deformation by short laser-induced pressure pulses, J. Fluid Mech. 828, 374 (2017).

[15] A. Klein, D. Kurilovich, H. Lhuissier, O. Versolato, D. Lohse, E. Villermaux, and H. Gelderblom, Drop fragmentation by laser-pulse impact, arXiv:1910.02522 (2019).

[16] C. Vernay, L. Ramos, and C. Ligoure, Free radially expanding liquid sheet in air: Time- and space-resolved measurement of the thickness field, J. Fluid Mech. 764, 428 (2015).

[17] Y. Wang and L. Bourouiba, Drop impact on small surfaces: Thickness and velocity profiles of the expanding sheet in the air, J. Fluid Mech. 814, 510 (2017).

[18] Y. Wang, R. Dandekar, N. Bustos, S. Poulain, and L. Bourouiba, Universal Rim Thickness in Unsteady Sheet Fragmentation, Phys. Rev. Lett. 120, 204503 (2018).

[19] S. Kooij, R. Sijs, M. M. Denn, E. Villermaux, and D. Bonn, What Determines the Drop Size in Sprays?, Phys. Rev. X 8, 031019 (2018).

[20] E. Villermaux and B. Bossa, Drop fragmentation on impact, J. Fluid Mech. 668, 412 (2011).

[21] G. Cisneros, J. S. Helman, and C. N. J. Wagner, Dielectric function of liquid tin between 250 and $1100^{\circ} \mathrm{C}$, Phys. Rev. B 25, 4248 (1982).

[22] F. E. C. Culick, Comments on a ruptured soap film, J. Appl. Phys. 31, 1128 (1960).

[23] D. Kurilovich, T. de Faria Pinto, F. Torretti, R. Schupp, J. Scheers, A. S. Stodolna, H. Gelderblom, K. S. Eikema, S. Witte, W. Ubachs, R. Hoekstra, and O. O. Versolato, Expansion Dynamics after Laser-Induced Cavitation in Liquid Tin Microdroplets, Phys. Rev. Appl. 10, 054005 (2018).

[24] J. A. Stratton, Electromagnetic Theory (McGraw-Hill Book Company, inc., London and New York, 1941), 2nd ed.

[25] N. Bremond and E. Villermaux, Bursting thin liquid films, J. Fluid Mech. 524, 121 (2005).

[26] A. Rozhkov, B. Prunet-Foch, and M. Vignes-Adler, Dynamics of a liquid lamella resulting from the impact of a water drop on a small target, Proc. R. Soc. Lond. 460, 2681 (2004). 
[27] J. Eggers, M. Fontelos, C. Josserand, and S. Zaleski, Drop dynamics after impact on a solid wall: Theory and simulations, Phys. Fluids 22, 1063 (2010).

[28] H. Lastakowski, F. Boyer, A.-L. Biance, C. Pirat, and C. Ybert, Bridging local to global dynamics of drop impact onto solid substrates, J. Fluid Mech. 747, 103 (2014).

[29] C. W. Visser, P. E. Frommhold, S. Wildeman, R. Mettin, D. Lohse, and C. Sun, Dynamics of high-speed micro-drop impact: Numerical simulations and experiments at frameto-frame times below 100 ns, Soft Matter 11, 1708 (2015). 